\title{
Masculinités hégémoniques et « sexualités » entre hommes au Maroc
}

Entre configurations locales et globalisation des catégories de genre et de sexualité

Hegemonic Masculinities and "Sexualities" Among Men in Morocco. Between

Local Configurations and Globalization of Gender and Sexual Categories

\section{Gianfranco Rebucini}

\section{OpenEdition Journals}

Édition électronique

URL : http://journals.openedition.org/etudesafricaines/17367

DOI : 10.4000/etudesafricaines.17367

ISSN : 1777-5353

Éditeur

Éditions de l'EHESS

\section{Édition imprimée}

Date de publication : 28 mai 2013

Pagination : 387-415

ISBN : 978-2-7132-2387-7

ISSN : 0008-0055

Référence électronique

Gianfranco Rebucini, « Masculinités hégémoniques et « sexualités » entre hommes au Maroc », Cahiers d'études africaines [En ligne], 209-210 | 2013, mis en ligne le 06 juin 2015, consulté le 16 juin 2020. URL : http://journals.openedition.org/etudesafricaines/17367 ; DOI : https://doi.org/10.4000/ etudesafricaines. 17367 


\title{
Masculinités hégémoniques et « sexualités » entre hommes au Maroc
}

\author{
Entre configurations locales et globalisation \\ des catégories de genre et de sexualité
}

C'est au printemps 2002 que je me suis rendu pour la première fois à Marrakech. C'était la première fois que j'allais au Maroc et c'était en même temps ma première expérience de travail de terrain. Au cours d'un aprèsmidi ensoleillé, je me trouvais sur la place Jemâa el Fna, essayant de concrétiser ce qu'est la tâche principale de l'anthropologue sur «son terrain »: l'observation participante. Installé à une terrasse de café, ou me promenant tranquillement, pour observer les «objets» de mon étude, en l'occurrence les hommes et les garçons dans l'espace public, je récoltais les données ethnographiques nécessaires pour mener à bien ma recherche sur la masculinité et les sexualités entre hommes au Maroc (à l'époque je finalisais un mémoire de DEA en anthropologie sociale). Je devais en effet conduire une analyse sur la masculinité au Maroc et dans le même temps chercher à comprendre comment les pratiques homoérotiques ou homosexuelles étaient éventuellement liées à la construction de cette masculinité. Je ne pouvais donc pas mener à bien ma recherche sans entrer en contact avec des hommes et garçons qui justement s'adonnaient à ces pratiques. Je savais déjà qu'en restant à proximité de la place Jemâa el Fna j'aurais plus de chances de faire des rencontres fructueuses en ce sens. Il ne fallut en effet pas longtemps pour qu'un garçon croise mon regard. Il me fit signe de le suivre. C'était la première fois que je me faisais racoler (ou que je racolais) dans la rue à Marrakech. Le garçon me « draguait » sans aucun doute. Il marchait devant moi, faisant attention à ne pas se faire remarquer par la police ${ }^{1}$. À ce moment-là, je me suis demandé : que faire ? Après un long moment d'hésitation, j'ai décidé de le suivre. Il était rentré dans un bar et monté à

1. Je n'ai su qu'après que la circonspection de sa part était due à une crainte de la «police touristique », très active à cette époque contre les faux-guides et aussi contre le racolage de prostitués et d'autres garçons sur la place Jemâa el Fna à Marrakech. 
l'étage. Nous nous sommes installés à une table et avons commencé à discuter. C'était mon premier contact avec un garçon marrakchi.

Mes expériences passées en Italie, et dans une moindre mesure en France, me disaient clairement que les intentions de Bader étaient à caractère sexuel. Une différence de taille quand même : en Italie et en France la drague en « plein air », dans l'espace public, se fait en général dans des lieux liminaux de la ville et le plus souvent la nuit ${ }^{2}$. Ici je me trouvais au centre-ville de Marrakech, au milieu de la foule, et en plein jour ${ }^{3}$ ! Comment ai-je fait pour reconnaître les intentions de Bader ? Avec quels moyens et quels présupposés ai-je compris ce qui était en train de se passer à ce moment-là ? À travers mes lectures, je savais que la plupart des garçons et des hommes dans les pays islamiques ${ }^{4}$, qui avaient des pratiques homoérotiques, n'étaient pas et ne se définissaient pas comme homosexuels. Je savais qu'il n'y avait pas de gay au Maroc ! À quoi donc pouvait correspondre cette «drague»? Quelle était la possibilité de partage et de compréhension d'un tel épisode qui finalement se terminait par une rencontre et qui donc avait fonctionné en termes de communication? Ne devais-je pas déjà interpréter cet épisode comme la confirmation d'une certaine universalité de l'identité homosexuelle, d'une caractérisation du sujet par sa sexualité, qui en dernière analyse nous avait permis, à Bader et à moi, de nous retrouver autour de cette table dans ce café ? J'aurais peut-être eu le droit et des raisons suffisantes pour inférer que Bader et moi partagions les mêmes valeurs et pour ainsi dire une même "culture homosexuelle », faite de codes de reconnaissance, d'attitudes corporelles, de gestes et de comportements qui découleraient finalement et universellement d'une même «nature psychologique », sinon «génétique ». Nous étions deux homosexuels ! Seule différence : l'un était Marocain et l'autre Italien. Tout pouvait donc être simple. La seule chose que j'aurais eu à faire, en tant qu'anthropologue, c'était de mettre en lumière les particularités des conditions d'existence de cette «nature psychologique »sous-jacente et partagée. Mais les choses se sont par la suite avérées plus complexes et problématiques que je ne l'avais imaginé et ressenti à ce moment de ma recherche.

Au début de mon travail d'enquête, j'étais donc partagé entre une vague connaissance intellectuelle de la diversité des agencements sexuels dans les

2. Voir à ce sujet B. PROTH (2002).

3. Pour une analyse des modalités de drague homoérotiques et homosexuelles masculines dans l'espace public à Marrakech je me permets de renvoyer à G. REBUCINI (2009, 2011).

4. Par «pays islamiques », j'entends ici les pays dont la religion majoritaire est l'islam, mais dont la culture majoritaire, l'Islam, et la société ne se réduisent pas à cet élément religieux. Cette définition est de toute façon préférable à la plus galvaudée «pays arabo-musulmans » dont les deux termes tendent d'une part à se limiter aux pays de langue arabe et, d'autre part, à mettre l'accent uniquement sur le fait religieux. De cette façon, des pays non arabes comme la Turquie, l'Iran et l'Indonésie, ou des pays avec des fortes minorités non musulmanes comme Israël-Palestine, peuvent être considérés comme des pays islamiques. 
pays islamiques («il n'y a pas d'homosexuels»), et une perception assez claire d'une familiarité avec les pratiques, ne serait-ce que par rapport à la «drague ». C'est seulement après plusieurs mois de séjour au Maroc que j'ai commencé à me rendre compte de la différence intrinsèque des significations et des usages que les garçons et les hommes pouvaient adopter à partir de pratiques à l'apparence identique aux miennes. C'est en effet à la suite de plusieurs discussions et échanges que je me suis aperçu que les pratiques homoérotiques (drague, rapports corporels, affectivité) ne se cristallisaient pas autour d'une identité et d'une psychologie particulière, et ne s'inscrivaient donc pas dans la manière dont j'appréhendais habituellement l'homosexualité. Il pouvait y avoir des pratiques érotiques tout à fait similaires aux miennes sans que pour autant les personnes impliquées se définissent ou soient définies comme homosexuelles ou gay.

Au début de mon séjour à Marrakech, je me trouvais face à une incompréhension fondamentale des catégories locales. Il me fallait me remettre en question, et dans le même mouvement remettre en question les catégories d'analyse qui jusqu'alors ordonnançaient le discours et la connaissance de mon expérience, notamment sexuelle. Cette remise en question a été aussi l'aboutissement d'une série de questionnements éthiques, résultant du travail inquiet et d'expériences contradictoires pendant le terrain, qui peuvent difficilement être éludés. Dans le cas précis d'une étude sur les masculinités et les pratiques érotiques entre hommes au Maroc, il est essentiel de se poser les questions catégorielles et politiques «qui est qui ? », « qui est quoi ? , mais aussi et surtout «qui est quoi pour qui ?». Ainsi, une analyse des pratiques érotiques entre hommes qui se servirait d'une épistémologie sexuelle ${ }^{5}$ fondée uniquement sur l'homosexualité identitaire, non seulement effacerait tout un éventail de questions en réduisant les différentes subjectivités et pratiques corporelles présentes au Maroc à un seul et unique modèle, mais cette simplification pourrait aussi répondre à un dessein épistémologicopolitique universaliste et eurocentré dont les résultats viendraient, par ricochet, confirmer la validité de cette épistémologie particulière qui se veut et se voit comme universelle ${ }^{6}$. C'est pour cela que le travail ethnologique requiert une «ouverture » du sujet connaissant qu'est l'anthropologue. À cet égard et dans des termes plus généraux, Donna Haraway (2009 : 335)

5. Je reprends ici la formule «épistémologie sexuelle » comme elle est introduite dans le travail de Joseph MASSAD (2007). Cet auteur, en prolongeant la pensée de Michel Foucault, utilise "épistémologie sexuelle » pour essayer de différencier l'ensemble des catégories, des théories et des pratiques discursives et corporelles qui constituent les conditions de possibilité et de connaissance de deux modèles distincts de sexualités. L'épistémologie sexuelle occidentale moderne, telle qu'elle a été aussi décrite par Michel FoucAult (1976) dans La volonté de savoir comme un « dispositif», peut se distinguer donc d'une épistémologie sexuelle «arabe » de la période pré- et, en large partie, postcoloniale, décrite par J. MASSAD (2007) dans Desiring Arabs.

6. Voir à ce sujet S. P. Mohanty (2001). 
propose la mise en place d'une nouvelle objectivation qui reposerait sur le dépassement de la dualité foncière de la pensée occidentale entre sujet et objet, en prônant une épistémologie « localisée » et « située » qui a le mérite d'encadrer l'analyse des rapports de pouvoir dans une «perspective partielle ». Ainsi, pour cet auteure,

«Seules les perspectives partielles valent promesse de vision objective. [...] Les récits de la culture occidentale sur l'objectivité sont toujours des allégories du traitement idéologique des rapports entre ce que nous appelons esprit et corps, distance et responsabilité, tous profondément inscrits dans la question de la science telle qu'elle intéresse le féminisme. L'objectivité féministe concerne des localisations délimitées et des savoirs situés, pas la transcendance et la coupure entre le sujet et l'objet. »

De surcrôt, c'est justement à partir d'une analyse du sujet, qui n'est pas un sujet indivisible mais multiple, multidimensionnel, hétérogène, qu'une vraie objectivation deviendrait possible. De fait, si la connaissance scientifique ressemble métaphoriquement à l'acte de la vision, comme le suggère D. Haraway (ibid. : 339-340) dans le même texte, et si la vision ne peut exister que par la collecte des différences, alors un sujet multiple et composite détient une position privilégiée dans la démarche d'objectivation scientifique. En fait, poursuit l'auteure, le sujet connaissant,

«[le soi sachant] est partiel sous tous ses aspects, jamais fini, jamais entier, ni simplement là et original ; toujours construit et cousu de manière imparfaite, il est de ce fait capable de s'unir à un autre, de voir avec lui sans prétendre être l'autre. C'est à cela que tient la promesse de l'objectivité. »

Nous voyons ici clairement comment ce «voir avec un autre sans devenir l'autre » et cette identité hétérogène et construite sont propres aussi à l'anthropologue sur le terrain à condition de toujours garder cette position critique d'ouverture et de positionnement, sans prétendre à la transcendance indifférenciée du sujet connaissant.

Cette question du sujet connaissant de l'anthropologue est strictement liée aux questions qui concernent la construction des catégories par lesquelles le chercheur interprète ou, mieux, comprend, les réalités sociales qu'il analyse. En effet, si le sujet connaissant est toujours partiel dans son ontologie comme dans sa capacité à la connaissance, les catégories utilisées, ainsi que les conditions de possibilité et d'utilisation de ces catégories dans le jeu de classification et de reconnaissance mutuelle, demeurent problématiques. Ainsi, revenant à l'exemple ethnographique décrit précédemment, il n'est pas du tout sûr que la reconnaissance du jeu de la «drague » doive se faire, comme je l'ai dit, du seul côté de l'anthropologue qui est en train d'analyser la situation. Bader aussi s'investit dans ce jeu d'utilisation de catégories (conventions sociales, postures corporelles, attitudes et gestes socialement construits) et dans un jeu de connaissance et de reconnaissance. 
En clair, si la rencontre a été possible, c'est aussi par le travail cognitif et émotionnel de Bader qui, probablement, connaissait les codes et les conventions de la «drague » d'un Européen. Pour arriver à ses fins, il a forcément dû mobiliser un ensemble d'agencements conscients et inconscients, intellectuels et corporels qui, d'une part, étaient déjà là grâce à son expérience passée et, d'autre part, se reconfiguraient dans le moment même de l'interaction et du processus du fait social de la «drague». On touche ici à une vision complexe des interactions sociales qui ne sont pas simplement et déjà là, mais qui se forment et se reconfigurent incessamment et sous le signe d'un processus d'agencement continuel sur différentes échelles. Dans cette perspective, il me paraît alors que la question de la reconnaissance (des codes, des attitudes, etc.) ne peut pas être abordée d'un seul côté de la relation. En ce sens, l'anthropologue reconnaîtrait ce qui se met en place comme relation et interaction sociale, et il «déciderait » que l'interaction et l'expérience qu'il est en train de vivre est d'une nature ou d'une autre, ou a une signification ou une autre. À l'inverse, il me semble que c'est dans la relation que l'interaction prend sa signification et tout son sens. Et cette relation est faite et se déroule de telle sorte que l'anthropologue ne peut pas prétendre à une vision d'en haut ou de loin. Il est déjà et complètement pris dans cette relation complexe. De surcroît, parce que l'interaction est pétrie d'un ensemble de relations de pouvoir, déjà là, la reconnaissance comme instrument de connaissance doit prendre en compte, et au sérieux, les mots, les représentations et les affirmations des individus concernés. C'est pourquoi la question de la prise en compte des catégories locales ne doit pas être seulement l'occasion d'une traduction (au sens étymologique de «traverser, passer d'un point à l'autre ») vers nos propres catégories d'analyse, selon le schéma classique de l'anthropologie comme science de traduction et d'interprétation. La tâche de l'anthropologue est plutôt de prendre à la lettre les catégories locales, et d'admettre que peut-être il n'y a pas de «traduction» possible, et que cela n'empêche en rien une réelle compréhension de l'utilisation et de l'usage qui sont faits de ces catégories par les individus dans la vie quotidienne.

Cette question de l'usage des catégories et de la possibilité de la conscience de cet usage de la part de l'anthropologue demeure de la plus grande importance, et cela pour au moins deux raisons. D'une part, la connaissance de cet usage étant toujours partielle, l'anthropologue doit s'imposer une certaine modestie dans ses affirmations, notamment quand il manie des catégories qui décrivent des situations et des expériences aussi mouvantes et instables que les pratiques érotiques, en l'occurrence entre hommes. Et, d'autre part, parce que, comme l'a remarqué Leslie Feinberg (2006), l'attention portée aux catégories et aux identités sexuelles dans les pays islamiques comme aux sujets non blancs en Europe, peut aussi être le résultat d'un contexte géopolitique global marqué par une forte pression islamophobe. Les travaux récents de Jasbir K. Puar (2007), de Joseph Massad 
(2007) et de Jin Haritaworn $(2008)^{7}$ montrent en effet à différents titres et avec des accents divers, que les questions de sexualité, et notamment d' « homosexualité » masculine, ainsi qu'une certaine vision des masculinités dans les pays islamiques, deviennent de plus en plus problématiques.

\section{Deux modèles épistémologiques. Une cartographie}

Il est intéressant à ce propos de se pencher sur l'articulation et la frontière entre le zamel et le gay, deux figures emblématiques au Maroc. Cette analyse et le travail de cartographie qui l'accompagne n'a pas pour objectif la définition de catégories identitaires autoréférentielles, mais plutôt l'éclaircissement de lignes de frontière perméables entre deux modèles épistémologiques que j'appellerai ici «genre/identités sexuelles » et « genre/pratiques érotiques » et qui coexistent au Maroc même s'ils sont le plus souvent incommensurables. Cette cartographie permet de mettre en contexte les expériences et les négociations des garçons et des hommes à Marrakech en rapport à leurs pratiques érotiques et affectives.

\section{Généalogies et contours de l'épistémologie gay}

Dans son importante analyse, l'historien John D'Emilio (1993) montre que les identités gay contemporaines seraient le résultat de l'évolution du capitalisme industriel du XVIII ${ }^{\mathrm{e}}$ siècle. Avec l'expansion du capital et la montée du travail salarié, à partir de cette époque, on a vu se produire des changements radicaux en rapport avec la structure et les fonctions mêmes de la famille, avec l'idéologie sous-tendue par cette institution, et avec la signification des relations sexuelles à l'intérieur comme à l'extérieur de celle-ci. Pour cet historien, la force du capitalisme industriel a été de créer les conditions d'une séparation toujours plus forte entre une sexualité de procréation et une sexualité de plaisir. Puisque les individus s'affranchissaient des liens familiaux (à cause de leur entrée dans le marché du travail), le capital a également permis que les individus organisent leurs pratiques érotiques d'une façon indépendante de la reproduction, notamment concernant les pratiques entre personnes du même genre. Pourtant, d'autres auteurs ont aussi montré qu'une vision uniquement économique du capitalisme ne pouvait, à elle seule, expliquer tous les changements historiques qui ont caractérisé l'avènement d'une identité homosexuelle. Ainsi, l'identité lesbienne a plutôt été le produit d'un changement du système de genre. En fait, c'est le bouleversement de la division sexuée du travail au $\mathrm{Xx}^{\mathrm{e}}$ siècle qui a permis aux femmes des classes moyennes de s'affranchir de la famille, en devenant

7. Voir aussi J. Haritaworn, T. TAuqir et E. ERdem (2008). 
indépendantes financièrement et en acquérant aussi une autonomie personnelle à l'extérieur de la famille et par rapport aux hommes (Gluckman 1997). Les raisons historiques de l'avènement de l'identité homosexuelle ne se situeraient donc pas uniquement dans une histoire purement économique du capitalisme comme le pense D'Emilio. D'autres auteurs mettent plutôt l'accent sur une histoire des idées, ou si l'on veut sur une histoire culturelle et idéologique de l'Occident et notamment des États-Unis. Ainsi, comme le fait remarquer l'historien David Halperin (1990: 31), à la suite des travaux de George Chauncey (1994) sur l'histoire culturelle de New York, c'est à partir du XX $x^{\mathrm{e}}$ siècle qu'une nouvelle taxinomie des pratiques sexuelles s'est détachée d'une catégorisation par le «genre » pour se fonder uniquement sur le sexe anatomique des participants à l'acte.

C'est donc cette disjonction de la sexualité et du genre des partenaires qui permet désormais idéologiquement, mais aussi très pratiquement, de considérer et de traiter les hommes ayant des rapports sexuels avec d'autres hommes, non plus en fonction de leurs pratiques, mais de manière globale en y incluant tous les hommes, qu'ils aient une position réceptive ou insertive, dans une même identité homosexuelle. Ce glissement dans la catégorisation des pratiques érotiques, d'une classification par genre à une classification par sexualités, a alors permis, en Occident, l'apparition d'une masculinité particulière qui, dorénavant, ne devait plus seulement s'opposer au féminin, mais se construire aussi en opposition à l'homosexuel. Comme le dit l'historien George Mosse (1997 : 17-18), la construction de la masculinité moderne occidentale s'est faite historiquement en fabriquant des identités négatives du masculin, comme l'homosexuel, mais aussi le juif, le prolétaire, le criminel, et le colonisé ${ }^{8}$. Ainsi, cet auteur affirme que «la virilité moderne avait besoin de ses contretypes, mais, en retour, ceux qui se voyaient ainsi caractérisés ont tenté, soit d'imiter le type dominant, soit de se définir en opposition à lui. Nul n'a complètement échappé à son emprise ». L'épistémologie «genre/identités sexuelles » de l'Occident moderne s'est ainsi construite sur la dichotomie identitaire hétérosexuel/homosexuel. En ce sens, l'apparition de la catégorisation sexuelle et l'émergence de la dichotomie homosexualité/hétérosexualité se sont faites par la construction d'un système d'imbrication du genre et des identités sexuelles. Et pourtant, l'identité gay semble être quelque chose de différent de la seule identité homosexuelle. L'identité gay peut être définie comme faisant partie de ce que Barry Adam (1990) appelle une «homosexualité moderne ». Selon cet auteur, c'est à partir des années 1970, et notamment après les événements de Stonewall à New York en 1969, que l'on formalise cette nouvelle organisation discursive. Les caractéristiques de l'homosexualité du « gay moderne » sont alors les suivantes :

8. Voir aussi sur ce point E. DoRLIN (2006). 
«1. Les relations homosexuelles ont été capables de s'échapper de la structure du système dominant de la parenté hétérosexuelle. 2. L'homosexualité exclusive, maintenant possible pour les deux partenaires, est devenue une voie alternative aux formes conventionnelles de la famille. 3. Les liens intra-genrés ont développé des nouvelles formes sans se structurer autour de catégories particulières concernant l'âge ou le genre. 4. Les personnes ont commencé à se découvrir mutuellement et à former des réseaux de sociabilité à large échelle non seulement grâce aux relations sociales existantes mais aussi grâce à leurs intérêts homosexuels. 5. L'homosexualité est devenue une formation sociale en soi, caractérisée par une conscience de soi et une identité de groupe » (ibid. : 24).

À partir des années 1970, comme le souligne Manalansan IV (2003 : 23-24),

«L'identité gay est devenue bien plus que l'homosexualité, le désir pour une personne du même genre, et des pratiques sexuelles. Durant les trois décennies depuis Stonewall, il est devenu évident que l'identité gay a signifié toutes ces choses ensemble et encore plus. [...] La montée d'institutions culturelles, d'images et de pratiques a formé l'idée d'une culture gay unique qui interfère avec d'autres systèmes culturels aux États-Unis. »

En d'autres termes, on pourrait dire que, dans les sociétés occidentales, les identités gay se caractérisent par le fait de former un système culturel parmi d'autres qui se différencie par des traits culturels et discursifs qui lui sont propres. Parmi ces traits, on distingue un assez fort accent placé sur une conception individualiste de la personne (coming-out, prise en charge de soi, empowerment), une anxiété vis-à-vis des relations familiales (la famille traditionnelle est souvent considérée comme une entrave au bon déploiement de l'identité gay; c'est normalement au sein de la famille que le comingout est censé être le plus problématique ; c'est aussi le départ de la sphère familiale qui est censé être le point initial d'une trajectoire identitaire gay), et surtout par un discours sur une essence psychologique intérieure stable et fixe (l'identité gay comme orientation sexuelle exclusive). Il semble bien que ces différents traits se comportent comme des éléments d'un récit général qui prend la valeur d'une ontologie. L'ontologisation de ce récit en est aussi une naturalisation, et une universalisation. Ce récit, pour qu'il soit vrai, crédible, fondé, se doit d'être universel (le caractère psychologique et essentialiste n'est jamais trop loin). Pour paraphraser Katie King (2002 : 41-42) qui écrit sur l'identité lesbienne, on pourrait résumer ce récit comme suit : 1. l'identité gay n'est pas une phase passagère ; 2. les gay ne singent pas les hétérosexuels; 3 . les bisexuels sont des gay désorientés.

Ce récit, qui est bien sûr situé dans un contexte culturellement et historiquement particulier, est répandu et reproduit à une échelle globale par les moyens de diffusion de la culture euro-américaine. Le cinéma, la musique, les moyens d'information, la publicité, la pornographie gay répandent cette vision de l'identité gay bien au-delà de l'aire culturelle occidentale. La force des nouveaux moyens de communication de masse, notamment l'Internet, 
accélère ces circulations. C'est donc à ces mêmes éléments narratifs que les hommes et les garçons au Maroc se trouvent confrontés. Il est important de remarquer, pourtant, que ces transferts et circulations ne se font jamais de façon neutre, et à sens unique. Il est toujours question d'articulations, de réélaborations et de reconfigurations de ces éléments dans un cadre local de réception.

\section{Cartographie historico-sociale des pratiques érotiques entre hommes}

Le Maroc et plus en particulier la ville de Marrakech ont été historiquement décrits comme une terre vouée de longue date à l'homosexualité et comme un lieu où les catégories sexuelles se brouillent. Comme Edward Saïd (1978) l'a montré, les catégories de l'orientalisme tendent à construire, redéfinir et reproduire une géographie imaginaire et des représentations sur l'Orient. Les éléments de cette géographie sont encore aujourd'hui des catégories utilisées, de façon cachée ou manifeste. La question du savoir historique et des études littéraires sur lesquels sont construites ces mêmes catégories demeure un aspect fondamental dans l'étude de la sexualité et des pratiques homoérotiques au Maroc.

Différents travaux traitent du sujet : ils sont tantôt l'œuvre de spécialistes d'études arabo-islamiques qui ont cherché à donner une vision d'ensemble, tels Dunne (1990), Schmitt (1992) et Abukhalil (1993); tantôt le travail de non spécialistes tels Greenberg (1988), Murray et Roscoe (1997), Murray (2000) qui s'attaquent au sujet dans des chapitres d'ouvrages dédiés à la civilisation islamique en général dans une démarche comparative et historique ; tantôt des remarques isolées dans des travaux qui s'intéressent à des sujets plus larges, comme dans les cas de La sexualité en Islam de Abdelwahab Bouhdiba (1975) ou d'une œuvre arabe ou persane particulière dans l'espace de la critique littéraire (Wright \& Rowson 1997). Tous ces travaux, d'une façon ou d'une autre, reposent sur l'idée qu'il existe quelque chose que l'on pourra nommer «homosexualité » dans les sociétés islamiques, quelle que soit l'époque ou la position géographique — ou du moins esquiventils toute problématisation à ce sujet. L'étude de Khaled El-Rouayheb (2010), en revanche, questionne l'idée de l'existence d'une homosexualité dans ces sociétés. En se basant sur des sources de provenance géographique différente de la période précoloniale sous l'Empire ottoman, l'auteur montre que pendant cette période historique on ne peut pas parler d'homosexualité en tant que catégorie identitaire.

D'un point de vue historique, comme le souligne Frédéric Lagrange (2008 : 125), dans les sociétés islamiques, jusqu'à l'époque moderne,

«Les sens des termes liwât (sodomie) et lût dans leur acception juridique et littéraire. [...] Le fa'il fi'l qawm lût ("celui qui commet l'acte du peuple de Loth") du juriste est tout homme se livrant, comme 
partenaire actif ou passif, à une pénétration anale. Pour le littérateur, le lûtî ne saurait être que le partenaire actif; de plus, le terme connote une préférence et un comportement répétitif, et non comme pour le juriste un acte susceptible d'être isolé. »

Cette différence sémantique est, selon l'auteur, la conséquence de la construction d'un personnage littéraire de "l'homme adonné à la course aux éphèbes, jouant toujours le rôle insertif » (ibid. :126). Dans la tradition juridique pré-moderne, n'ayant pas une base coranique attestée (les termes liwât et lûtî n'existant pas dans le Coran), le liwât a donné lieu à des débats importants selon qu'il était apparenté au zinâ (fornication, adultère) ou assimilé à l'abomination du peuple de Loth (dont on trouve en revanche l'histoire dans le Coran). Quoique la loi du fiqh ait reconnu les deux partenaires en tant que lûtî, c'est-à-dire pratiquant le liwât, le débat s'est aussi focalisé historiquement sur la différenciation ou non du traitement du pénétrant et du pénétré.

Le partenaire réceptif pouvait échapper à la responsabilité de ses actes dès lors qu'il était considéré comme souffrant de ubnah (maladie affectant un individu qui le rendait désireux d'être pénétré) ou sous la contrainte d'un maître, ou à la recherche d'un gain matériel, en excluant dans ce cas tout désir sexuel volontaire. Selon Lagrange (ibid. : 128-129),

« Le partenaire passif, à moins qu'il ne s'agisse du pathologique ma'bûn, n'est pas présumé rechercher une satisfaction sexuelle dans la relation anale [...]. Il y a là une tension au sein même des discours juridiques entre une logique "égalitaire" où les deux partenaires doivent être également punis, et une logique "discriminante", qui peut soit disculper le partenaire passif, moins responsable [...], soit au contraire l'estimer condamnable dans tous les cas, comme chez certains hanbalites. »

Pourtant, ces débats juridiques, qui se sont prolongés au cours des siècles jusqu'à l'introduction d'un droit positif, même s'ils informent sur l'intérêt que le fiqh a toujours porté à la question du liwât, doivent être relativisés par les procédures effectives qui pouvaient conduire à l'inculpation ou non d'un individu. Comme dans le cas du zinâ, pour que le délit puisse être pris en considération par le juge et qu'un châtiment soit possible, la présence de quatre témoins oculaires ou d'un aveu non rétracté était nécessaire. Ainsi, la procédure d'inculpation nécessitait une publicité et même un caractère scandaleux pour qu'elle soit prise en compte. C'est pourquoi « dans le cas de liwât comme dans les cas de sodomie, le juriste remercie Dieu de pouvoir cacher les vices des croyants qui ne sont pas ostentatoires dans leur transgression de la loi divine » (ibid. : 129). On voit ainsi que les cas de transgression étaient généralement traités avec la plus grande discrétion et en dernier recours, ou en guise de bonne gouvernance, pour faire un exemple.

Il en va différemment à l'âge de la colonisation.

« Le XIX ${ }^{\mathrm{e}}$ siècle fut un moment d'adaptation des lois positives à des nouvelles réalités économiques et politiques et, dans une première phase, ce furent essentiellement 
des codes étrangers qui se virent appliqués dans le domaine pénal, quand bien même le droit familial demeure quant à lui largement référencé au fiqh» (ibid. : 132).

Dans le cas du liwât, le passage par l'adoption des systèmes juridiques coloniaux, qui condamnaient clairement les « actes contre nature », la sodomie et l' «homosexualité », et la plus récente réislamisation des codes pénaux des nations indépendantes ont aussi introduit un nouveau vocabulaire et une nouvelle épistémologie sexuelle. Ainsi, au Maroc, sont punis par la loi les « actes impudiques ou contre-nature avec un individu de son sexe » (art. 489 du 26 novembre 1962) ${ }^{9}$. Même si la loi ne fait pas référence à une identité homosexuelle en tant que telle, les définitions d' "actes contre-nature » et d' « individu de son sexe » bouleversent l'épistémologie sexuelle du fiqh, qui faisait référence à la loi divine et non à un ordre de la nature, et traitait les individus dans leur spécificité en rapport à la position assumée dans les rapports sexuels, tandis que la loi marocaine ne fait plus du tout cette différence entre pénétrant et pénétré, pénétré consentant et pénétré contraint, passif par intérêt et passif pathologique, viol et relation consentante, laissant un générique «individu de son sexe » englober toutes ces catégories.

Selon Joseph Massad, les dichotomies sexuelles, normal/déviant, hétérosexuel/homosexuel, se seraient installées dans les pays islamiques à la suite de la colonisation et à travers le discours orientaliste occidental et nationaliste arabe. L'auteur localise une autre période-charnière de cette histoire coloniale en rapport aux catégories sexuelles : les revendications des mouvements pour les droits civiques des gay et des lesbiennes des années 1960 aux États-Unis et en Europe occidentale, qui se sont internationalisés à partir des années 1980 et 1990, ont participé à une hiérarchisation des réactions liées aux comportements et aux pratiques sexuelles entre civilisés et noncivilisés. C'est justement à cette époque d'intense activisme international de la part de ces associations qu'on observe l'émergence d'un changement de discours à la fois dans la sphère intellectuelle et dans le monde politique. L'auteur montre alors comment la question de l'homosexualité et son traitement par la voie de la répression montent dans les discours des mouvements radicaux islamistes naissants et dans le débat public, discours auxquels s'opposent les intellectuels arabes modernistes qui, d'une façon ou d'une autre, embrassent la cause de ces revendications. Il y aurait donc, à partir des années 1980 et 1990 , une forte « incitation au discours » ${ }^{10}$ pour catégoriser et distinguer des identités sexuelles qui, jusque-là, étaient restées pour le moins floues et à l'usage exclusif des classes favorisées des sociétés islamiques. On assiste dès lors aux mêmes changements de société et d'épistémologie, et avec les mêmes moyens de coercition de type policier,

9. L'article 489 du code pénal condamne «les actes contre-nature » à des peines allant de six mois à trois ans de prison ou à des amendes allant de 120 à $1200 \mathrm{Dh}$.

10. L'auteur reprend à son compte cette expression de Michel FouCAULT (1976). 
et d'assujettissement médical, qu'ont connu l'Europe et les États-Unis à partir du XIX siècle.

À ce propos, le scandale dit du «mariage homosexuel de Ksar el-Kebir » est des plus révélateurs. En novembre 2007, à Ksar el-Kebir, ville située à mi-chemin entre Rabat et Tanger, Fouad, du prénom donné dans la presse, un vendeur d'alcool à la sauvette, a décidé d'organiser une soirée privée dans une maison du quartier populaire de Hay Diwan, habituellement réservée aux célébrations de mariages. Le protagoniste aurait en effet eu une «vision », un rêve où il se serait vu en femme dans les habits d'une mariée (Achehbar 2007). Il a donc voulu reproduire ce rêve dans la réalité, en organisant une fête ayant les caractéristiques d'un mariage, sans qu'il ait pour autant un marié à ses côtés, avec une procession dans les rues du quartier, à laquelle ont participé plusieurs dizaines de personnes. Des enregistrements vidéo de la célébration ont été ensuite diffusés sur l'Internet ${ }^{11}$. Deux jours après, les sections locales du parti islamiste PJD ${ }^{12}$ et du mouvement Al Adl Wal Ihsane ${ }^{13}$, ainsi que différentes associations ont lancé une pétition pour demander aux autorités judiciaires de la ville l'ouverture d'une enquête officielle sur la célébration d'un «mariage homosexuel». Face au refus d'ouvrir l'enquête, ces mouvements ont organisé des manifestations avec des milliers de personnes, essayant de prendre d'assaut la maison de Fouad qui a échappé de peu à un lynchage en se livrant à la police. La presse $^{14}$, surtout celle qui est proche du PJD, a amplifié les faits en dénonçant à la Une les événements comme un authentique mariage homosexuel, montrant même un échange de bagues ${ }^{15}$. Les autorités, après avoir tenté de nier

11. Sur le site d'échange de vidéos <http://www.youtube.com>.

12. Parti marocain à référence islamique, le PJD (Parti de la justice et du développement) s'inscrit dans le cadre de la monarchie constitutionnelle, reconnaît le fait que le roi du Maroc est également Commandeur des croyants, et il est représenté au Parlement par 46 députés sur un total de 325, lors des élections législatives de septembre 2007. Il reste dans l'opposition jusqu'aux élections législatives de 2011 où il devient le premier parti au Parlement avec ses 107 députés. À la suite de ces dernières élections, le Roi Mohamed VI a nommé Abdel-Ilah Benkirane, leader du PJD, chef du gouvernement.

13. Littéralement Justice et Spiritualité, Al Adl Wal Ihsane est un mouvement islamiste marocain, non légal mais toléré par les autorités. Il a été créé en 1973 par Abdessalam Yassine. Il ne reconnaît pas le roi comme Commandeur des croyants. La fille de Yassine, Nadia Yassine, principale porte-parole du mouvement, au Maroc comme à l'étranger, suite à ses déclarations en 2005 sur le fait que le meilleur système politique pour le Maroc serait la République, a subi une procédure judiciaire pour «atteinte aux institutions sacrées de la nation», mais qui n'a pas encore abouti à un vrai procès.

14. Le quotidien arabophone Al Massae, un des meilleurs tirages de la presse marocaine, a, notamment, publié des photos du dit «mariage » à la Une, quelques jours après les faits, ce qui a été un des facteurs principaux du déclenchement des émeutes.

15. La principale revue du PJD, Attajdid, a, en fait, publié des photographies montrant une main d'homme passer une bague au doigt d'un autre homme, sans que l'on puisse en voir les visages, nommant pourtant en légende Fouad comme sujet de l'image. 
le caractère «homosexuel ${ }^{16}$ de la célébration, ont cédé aux pressions de l'opinion en arrêtant huit personnes dont Fouad, qui a été jugé et condamné à une peine de dix mois de prison ferme assortie d'une amende de 1000 dirhams (Hamrouch 2007), pour « commerce illégal d'alcool » et « perversion sexuelle » en violation de l'article 489 du Code pénal utilisé dans les cas de sodomie et d'actes homosexuels en flagrance de délit.

Le fait qu'une telle célébration, relevant plutôt d'une performance de travestissement, ait pu être dénoncée comme un mariage homosexuel en bonne et due forme par les mouvements islamistes, démontre ainsi d'une part que les représentations de l'homosexualité identitaire de type occidental ont une emprise de plus en plus décisive sur l'épistémologie sexuelle marocaine et, d'autre part, que ces représentations tendent à recouvrir tout le champ des interprétations possibles dans les différents comportements liés aux rapports de genre. Les pratiques de travestissement, qui demeurent d'une banalité quotidienne, prennent une toute autre signification dès qu'elles sont associées, même si c'est de façon abusive, au mariage. Il est aisé de comprendre le transfert fait par des mouvements d'inspiration islamiste, entre le travestissement et l'homosexualité masculine, dans un contexte plus international où la nouvelle frontière des luttes des mouvements gay et lesbiens d'inspiration libérale demeure justement le «mariage homosexuel ». Un tel télescopage, qui n'est sûrement pas le fait du hasard, s'insère donc dans une dialectique globale du discours de la modernité et de la tradition dont les mouvements de défense des droits de l'Homme d'un côté et les mouvements islamistes de l'autre se veulent les porte-paroles, partageant de ce fait les mêmes catégories et les mêmes taxinomies sexuelles.

\section{Pratiques homoérotiques versus homosexualité identitaire et leur rapport aux masculinités hégémoniques}

Dans la situation du Maroc d'aujourd'hui, et plus particulièrement dans le contexte urbain de la ville de Marrakech, les catégories d'hétérosexualité et d'homosexualité font désormais partie du système idéologique qui, d'une façon ou d'une autre, produit et reproduit les représentations sur la sexualité et le genre. Les différentes pratiques deviennent de la sorte licites ou illicites selon cette nouvelle épistémologie sexuelle et de genre. Mais cette épistémologie ne s'est pas greffée simplement à la place de l'ancienne. Des différences importantes peuvent être, en fait, retrouvées entre les différentes classes composant la société marocaine. Notamment dans le milieu urbain, les classes favorisées et généralement instruites partagent les catégories

16. Le ministre de l'Intérieur de l'époque, Chakib Benmoussa, le 28 novembre 2007, en intervenant dans une réunion de la commission de l'Intérieur au Parlement déclarait en effet : «Cette fête est liée à des rituels de charlatanisme » (BERNICHI 2007). 
sexuelles occidentales, tandis que les classes subalternes demeurent dans une attitude plus complexe vis-à-vis de ces catégories et de la masculinité notamment.

Il est possible d'analyser les formes locales de masculinité sur un axe de gradation allant du plus masculin, la masculinité hégémonique (Connell 1995 $)^{17}$ ou idéale, à un toujours moins masculin, correspondant à toutes les formes de masculinité non hégémoniques. Il apparaît alors concevable de considérer une pluralité de masculinités. Ces masculinités se construisent et se donnent toujours à voir dans un sens relationnel, complexe et fluide. Dans ce sens, masculinité et féminité sont toujours pensées en relation. Cela veut dire que dans la définition d'un degré de masculinité (plus ou moins forte, plus ou moins accomplie, plus ou moins hégémonique) la place de la «féminité » demeure un terme fort du nuancier. Dans cette perspective, la «féminisation» est le signe d'une moindre masculinité, mais pas de son manque absolu, ni de son opposé ontologique. La féminisation et la subalternité à une masculinité hégémonique doivent toujours être vues comme des moments et des positions précaires, tout comme la masculinité hégémonique d'ailleurs. Les positions relatives entre les masculinités dépendent ainsi de plusieurs facteurs socio-économico-culturels qui non seulement peuvent changer au cours de la vie d'un individu (selon l'âge, le statut, la position sociale), mais qui changent aussi synchroniquement dans des contextes de pouvoir différents. Une « féminité masculine » ne signifie donc pas et jamais le basculement de la position sociale d'un homme dans une altérité absolue qui serait «le monde des femmes» ou la féminité. Au contraire, le jeu complexe des masculinités s'actualise et se déploie, pour l'essentiel, dans un monde d'hommes. Ainsi, ce sont des qualités comme l'indépendance économique, l'autonomie décisionnelle, la maîtrise des affects et des émotions, ainsi qu'une certaine capacité à «faire face » vis-à-vis des autres hommes, une mise en scène de compétences liées au corps et à la force physique, telle la violence et les prouesses, et une relation de pouvoir hiérarchique par rapport aux femmes, qui déterminent la qualité de la masculinité hégémonique et le discours à travers lequel elle émerge. Dans ce cadre discursif, la mobilité, l'activité, la force, la sagesse, le discernement ('aql) de l'homme s'opposent ainsi à la lenteur, l'immobilité, le désordre, la faiblesse et une moindre faculté de jugement de la femme. Ce sont donc ces qualités et attitudes qui, distribuées de façon différentielle et avec plus ou moins d'importance, déterminent le degré de masculinité d'un individu. Dans cette perspective, parce que ces qualités et attitudes ne sont jamais complètement acquises ou présentes au même temps, nous devons constater que la masculinité hégémonique idéale ne peut être qu'une place vide ${ }^{18}$

17. Pour une révision et une réactualisation récente de ce concept voir R. W. ConNELL et J. W. Messerschmidt (2005) et R. Howson (2006).

18. Pour une analyse fine de l'hégémonie comme «place vide» voir E. LACLAU (2000). 
(parce que jamais atteinte) dans le jeu complexe des attributions et des assignations de masculinité.

Dans le cadre de la définition locale de la masculinité hégémonique, le fait d'être actif et pénétrant demeure une caractéristique fondamentale, bien que non suffisante. De ce fait, l'opposition rajul/zamel, c'est-à-dire homme/ moins-homme, recouvre, dans les représentations de soi, la dichotomie des plaisirs sexuels pénétrant/pénétré. Cette opposition n'est pourtant pas opératoire d'une façon exclusive dans les pratiques réelles et dans la mise en acte des plaisirs corporels. Ainsi, Yassine, garçon de 19 ans du quartier populaire de Massira, avait l'aspect d'un jeune éphèbe quand je l'ai connu. Son corps était mince, presque maigre, son visage n'avait presque pas de barbe, et ses attitudes corporelles étaient plutôt «efféminées ». Il était étudiant, sans travail, un homme en formation, encore très loin d'une perspective de mariage qui le consacrerait en tant qu'homme. En outre, il cherchait souvent des partenaires plus âgés que lui, de préférence occidentaux. Il ne m'a jamais fait part d'un quelconque intérêt pour les femmes. Yassine pouvait donc être considéré, selon la définition marocaine, comme un zamel. Pourtant, quand je lui ai posé la question, il m'a dit qu'il aimait autant pénétrer que se faire pénétrer et que cela dépendait des occasions. Il avait eu différents amants déjà, la plupart plus âgés que lui, et il avait expérimenté avec eux la sodomie insertive et réceptive. Cela ne semblait pas l'étonner. Ce qui est important au Maroc, en effet, c'est la position publique, la représentation de soi en face des autres hommes. L'expression de la masculinité de la rajuliya se déploie dans l'espace public et non dans la chambre à coucher. C'est pourquoi, dans certaines circonstances, les hommes peuvent satisfaire leurs désirs d'un plaisir autrement stigmatisé, s'ils ont la conviction que tout cela restera dans le cadre d'une relation strictement intime.

Dans les chats de discussion sur l'Internet (utilisés à Marrakech aussi par les hommes issus des classes défavorisées, et où les échanges sont souvent très directs et dans un but purement sexuel), les mots pour indiquer les préférences sexuelles ne se disent généralement pas en arabe dialectal (darija), mais en français ou, plus rarement, en anglais. Ainsi, actif, passif, top et bottom servent de raccourcis dans une discussion qui, dans le cas d'un individu ne maîtrisant aucune langue étrangère, rentrerait dans les détails, en questionnant, par exemple, sur la possibilité de se faire pénétrer (thwini), de pénétrer ( $h w i)$ ou de se pénétrer mutuellement (ndirou nwiba), de se laisser faire une fellation (tmouss liya) ou de la faire (tmouss). Le mot zamel n'est jamais utilisé pour désigner une pratique spécifique, et il n'existe pas d'autres mots en darija pour désigner un individu fondamentalement actif, pénétrant, ou passif, pénétré. En arabe marocain, on nomme l'acte et non la personne. Ainsi, les termes français « actif » et « passif » ont, au Maroc, une signification toute relative. Ils ne désignent pas des individus, à proprement parler, mais plutôt des pratiques. Ils sont d'ailleurs strictement liés à la contingence du moment, aux envies, mais surtout aux statuts et à la 
représentation de soi des partenaires. Abdel, un garçon de 25 ans qui travaillait à l'époque comme expert informatique dans une entreprise privée, appartenant donc aux classes moyennes avec une formation supérieure et occidentalisée, à propos des affirmations sur les pratiques sexuelles respectives échangées sur les chats de discussion, me disait que : "Même si maintenant les gay arrivent facilement à déclarer qu'ils aiment être enculés, sincèrement d'après mon expérience, plusieurs n'assument pas encore et disent qu'ils aiment enculer et, au moment de vérité, il s'avère qu'ils sont très passifs [...].»

Pourtant, cette volonté de cacher un désir de "passivité », quoiqu'elle exprime une crainte d'afficher publiquement un désir censé être dévalorisant pour la masculinité, ne signifie pas que les individus qui aiment se faire pénétrer se considèrent d'emblée moins masculins que les autres. Il y a certes une certaine féminisation dans le fait d'être passif, mais ceci, me semble-t-il, est dû plus au discours sur l'homosexuel en tant que « déviant », dans le sens occidental du terme, qu'à une réelle peur identitaire. Ce n'est pas la position dans la relation sexuelle qui décide du statut des partenaires mais plutôt le statut relatif des partenaires qui conditionne, sans exclusive, les rôles assumés pendant la relation sexuelle. Il y a là une fonction relative et non une identité. La différence entre la vision identitaire du rapport entre les dichotomies occidentales actif/passif et hétérosexuel/homosexuel et celle, plus imbriquée, du rapport entre pénétrant/pénétré et rajul/zamel au Maroc est donc une différence taxinomique. Elle s'actualise d'ailleurs plus particulièrement parmi les individus issus des classes populaires et subalternes. Si les hommes des classes aisées, qui sont plus en contact avec la culture européenne, assument plus ou moins complètement la vision identitaire et binaire occidentale, les individus des classes défavorisées semblent majoritairement la modérer à la faveur d'une dichotomie hiérarchique instable, analogique, homme/non-homme, ou moins-homme : l'opposition rajul/zamel.

Ainsi, c'est la dichotomie d' "espèce » actif/passif qui n'existe pas, et non pas seulement le terme "actif » en lui-même. Si, en effet, le mot zamel peut être traduit par passif, c'est seulement par un effet de fiction de traduction. Zamel est le donneur, celui qui donne son corps et qui, surtout, se met dans une position de dépendance, économique notamment (ce qui le rapproche momentanément d'une femme), et absolument pas un type particulier d'homme. Tout un chacun peut être, à un certain moment de sa vie, un zamel, personne n'est exclu de cette possibilité infamante. Cette passivité n'est donc pas une identité et de ce fait elle ne rejette pas l'individu vers le côté féminin. Il y a certes un déplacement sur une ligne qui verrait la masculinité hégémonique d'un côté et la féminité de l'autre, où le zamel se retrouverait déplacé vers le côté féminin, mais ce déplacement est tout relatif. Il est, d'une part, temporellement limité et, d'autre part, partiellement accompli. La masculinité, en fait, n'est pas quelque chose qu'on perdrait « en bloc» et pour toujours. 
Cette perte est, ainsi, partiellement identifiable à la perte de la face. La relation entre l'infamie de l' «homosexualité » du zamel et la perte d'honneur, que beaucoup d'auteurs ont décrite dans leurs analyses ${ }^{19}$, doit alors être reconsidérée à partir de là. La perte de l'honneur, pour les hommes, a beaucoup à voir avec une perte d'indépendance et la stigmatisation du zamel est, peut-être, beaucoup plus liée à cette perte, qui est d'ailleurs tout autant une perte relative de masculinité, qu'à un sentiment de rejet du désir homosexuel ou homoérotique. L' « actif » dans la relation n'est pas stigmatisé parce qu'il conserve tous les caractères de la masculinité : il est celui qui prend, et qui tient l'autre dans une situation de dépendance. Cela ne veut pourtant pas dire que l' «actif» ne désire pas le corps masculin de l'autre, qu'il soit le seul à pouvoir affirmer sa masculinité, et qu'il soit, de la sorte, « hétérosexuel » masculin. Cela veut dire seulement qu'il est, ponctuellement, du bon côté de la dichotomie hiérarchique. Il est aussi important de souligner que le zamel n'est pas entièrement l'équivalent du « passif » factuel de la relation. L'épithète de zamel, en fait, ne se réfère pas forcément à une pratique passive avérée, mais plutôt à une position sociale publiquement assignée. Est zamel celui que l'on qualifie ainsi. Le zamel est toujours un autre. Personne n'utilise ce terme pour définir une préférence personnelle envers une pratique sexuelle particulière comme la sodomie passive.

Au Maroc, cette juxtaposition entre la dichotomie actif/passif et l'hétérosexualité ne se fait donc pas du tout dans une perspective identitaire mais d'une façon pragmatique. S'il est un rapprochement entre le partenaire pénétré et la femme, ce n'est qu'accessoirement et temporairement, tant que la position du partenaire réceptif demeure ainsi. Il n'y aurait donc pas une « identification » mais plutôt une « féminisation » temporaire et contingente, un processus qui ne dit absolument rien sur l'identité et sur le caractère de l'individu.

De plus, si l'on regarde la question d'un point de vue sociolinguistique, le zamel, le mâle en quelque sorte féminisé, ne désignant pas une passivité de l'identité de l'homme mais plutôt son comportement contingent public et social, peut l'être, ou le devenir, selon les occasions de la vie quotidienne. Le zamel répond donc à l'homme, dans le sens de rajul, l'homme idéal et hégémonique, non en essence mais en intensité, en degré, comme dans une sorte de hiérarchie instable, parce que susceptible de fonctionner dans les deux sens à n'importe quel moment. De ce fait, rajul et actif ne sont pas du même ordre logique. Pour être un rajul, il faut certes être actif, mais pas seulement sexuellement, tandis qu'être actif sexuellement ne garantit en rien de posséder le statut de rajul, d'homme véritable. De plus, le fait d'être actif n'est pas l'exclusive des hommes. Les femmes aussi sont censées, dans des circonstances particulières, démontrer une attitude active et

19. À cet égard voir, entre autres, M. CheBEL (1988) et C. HAMEL $(2002,2003)$. 
entreprenante (Abu-Lughod 1986; Hilse Dwyer 1978 ; Kapchan 1996). À l'inverse, selon les circonstances, les hommes doivent faire preuve de déférence et de passivité, notamment dans les relations où ils se trouvent subordonnés ou dépendants (Hammoudi 2001). Activité et passivité, quoique idéalement connotés par le genre, ne se confondent donc pas avec la masculinité ou la féminité, comme le prétend la théorie psychanalytique de type freudien, mais participent à leur formation et construction dans la quotidienneté des rapports de pouvoir, y compris dans les rapports sexuels.

D'après certaines analyses des pratiques homoérotiques dans les pays islamiques, il n'y aurait pas une identité homosexuelle fixe, mais plutôt, selon les interprétations, une sexualité de substitution (Duran 1993), une homosexualité refoulée par le devoir de virilité (Schmitt 1992), ou une déviance sexuelle qui est « révolte contre Dieu » et son ordre naturel de la complémentarité qu'est le mariage (nikâk') (Bouhdiba 1975). Ces interprétations font l'impasse sur le rapport entre sexualité et système de genre. Les pratiques homoérotiques ne sont considérées qu'en tant que phénomène banal à l'intérieur d'un système de division masculin/féminin, et hétérosexuel/homosexuel, considéré comme allant de soi. La non problématisation du système sexe/genre/homoérotisme conduit ainsi toute analyse des pratiques homoérotiques présentes au Maroc à une impasse foncière.

Il apparait essentiel de problématiser les relations complexes entre les représentations sur le genre, la sexualité et les pratiques érotiques pour analyser la place que les pratiques homoérotiques occupent dans le contexte du Maroc d'aujourd'hui, et notamment dans la ville de Marrakech. Il est possible alors de noter des différences importantes entre les classes sociales dans la conception de comportements qui semblent être les mêmes et qui pourtant cachent des significations assez distinctes. Si, en effet, l'homosexualité identitaire est conceptualisée plus ou moins en des termes biologisants, médicaux et essentialistes, parmi les classes aisées de la société marocaine, ceci n'est pas sans rapport avec la construction d'un système de genre où les dichotomies masculin/féminin, et hétérosexuel/homosexuel travaillent ensemble et se soutiennent l'une l'autre. La masculinité et la féminité se situent alors à l'intérieur d'un système hétérocentré, dont le couple hétérosexuel demeure la pierre angulaire. Dans ce système, l'homosexualité masculine est assignée au rôle de terme de comparaison négatif d'une masculinité accomplie dans le mariage ou dans la relation hétérosexuelle exclusive. Si, comme le dit J. Massad, les pays islamiques ont, à partir du moment colonial et de manière plus décisive dans les dernières décennies, assimilé les catégories occidentales de la sexualité, notamment la dichotomie hétérosexuel/homosexuel, ils ne l'ont pas fait sans assimiler aussi le système de genre qui y est attaché.

Pourtant, la construction des genres au Maroc, notamment dans les classes populaires, se fait aussi, et dès les premiers instants de vie, par une nette différenciation entre les hommes et les femmes, et donc à travers une 
«genderisation» poussée, visant notamment le mariage. Cette caractéristique commune de la différenciation au sein d'un système hétérocentré, entre le Maroc et l'Occident, revêt cependant des significations différentes, parce que, dans le premier cas, l'assignation à un genre ne se fait pas en opposition à une identité sexuelle, mais plutôt en opposition avec l'autre genre. Un vrai homme serait celui qui se différencie le plus de la femme et non pas en même temps de l'homosexuel. De surcroît, la question essentielle n'est pas l'identité sexuelle d'un individu, mais le mariage et la procréation, qui n'ont rien à voir avec les pratiques sexuelles particulières. C'est donc la complémentarité des genres, de l'homme et de la femme, et la téléologie vers le mariage qui comptent dans cette technologie du genre ${ }^{20}$ marocaine.

Dans cette perspective, les garçons des milieux populaires que j'ai rencontrés à Marrakech, qui avaient des pratiques homoérotiques, et dont certains étaient clairement affichés en tant que zamel, ont tous affirmé la ferme volonté de se marier un jour. Leur jeune âge les mettait, pour le moment, à l'abri d'une telle éventualité, et pourtant le mariage demeurait pour eux un horizon d'accomplissement certain. Ils n'étaient guère effrayés par cette possibilité, mais au contraire ils la revendiquaient, aussi étrange qu'ait pu paraître cette revendication à mon esprit d'Occidental. En se plaçant dans une telle trajectoire, les garçons de Marrakech revendiquent leur condition proprement masculine, tout en affirmant qu'ils ne renonceraient pas pour autant à satisfaire leur désir homoérotique une fois mariés. Ainsi, Yassine, 19 ans, qui subissait une stigmatisation en tant que zamel, avait trouvé ma question sur l'éventualité d'un mariage bizarre, presque dénuée de sens. Il était normal pour lui de devoir se marier un jour. Le fait de vivre la situation inverse ne lui avait jamais traversé l'esprit. Il voulait, en fait, avoir des enfants et pouvoir construire une famille. Ce qu'il faisait dans sa vie privée n'avait pour lui pas de rapport avec son futur d'homme, et il ne pensait pas que ses activités actuelles, ou son «statut ${ }^{21}$ de zamel, pouvaient un jour conditionner sa construction définitive en tant qu'homme. L'absence totale de frustration et d'appréhension chez ces jeunes hommes dans leur vision en tant que futurs mariés démontre aussi que les pratiques homoérotiques, tant insertives que réceptives, ne préjugent en rien, selon leur jugement, de la réalisation d'une masculinité accomplie, qu'ils sont prêts à

20. J'utilise ici «technologie du genre » dans le sens donné par Teresa DE LAURETIS (2007 : 40-41) dans son célèbre article «La technologie du genre », selon lequel « le genre, comme la sexualité, n'est pas la propriété des corps ou quelque chose qui existe originellement chez les êtres humains, mais qu'il est "un ensemble d'effets produits dans les corps, les comportements et les relations sociales" [...] et ce grâce au déploiement d'une "technologie politique complexe" ".

21. J'utilise ici le mot de statut entre guillemets parce que Yassine ne partageait évidemment pas cet appellatif pour définir sa personne. Le mot zamel, on le rappelle encore une fois, est avant tout une insulte, auquel je fais jouer improprement ici et après le rôle d'identificateur d'un individu. 
assumer ultérieurement. Ainsi les plaisirs de la sodomie insertive ou réceptive n'ont qu'un lien assez faible tant avec la représentation de soi dans le domaine public qu'avec l'incorporation de la masculinité en tant qu'idéal.

La stigmatisation du zamel, dans ce cadre, prend alors une signification particulière. Le «stigmate » du zamel sert d'incitation au respect de la masculinité idéale, en marquant des frontières mouvantes entre un vrai homme, qui se veut toujours actif, indépendant, et mobile, et un zamel représenté comme ouvertement passif et dépendant. Ces frontières sont pourtant des frontières internes à la masculinité, comme des degrés d'intensité sur une échelle du masculin. Elles servent, ainsi, par le truchement de la création de cette catégorie excentrique qu'est le zamel, à délimiter un cadre précis de la masculinité. Le zamel, en négatif, sert l'homme à se définir. S'il n'y a pas de zamel, il n'y a pas non plus de «vrai homme », d'homme accompli. Le zamel n'étant pas une essence, une identité, une espèce, mais plutôt l'exemple d'une perte de masculinité, il ne représente pas un réel danger pour le système hétérocentré du genre au Maroc. La relative tolérance dont jouissent les pratiques homoérotiques s'explique ainsi par ce caractère réversible et mouvant de l'acquisition du statut d'homme, la masculinité pouvant toujours être réacquise, notamment par le biais du mariage. Tout est fait pour que l'ordre interne du genre soit respecté, mais les écarts sont toujours possibles, et la réintégration devient alors souhaitable. De ce fait, tant que le zamel demeure une catégorie transitoire et momentanée, et qu'il ne prétend pas à une identité fixe, à une individualisation marquée, mais accepte de se plier au système de genre et à la règle hétérocentrée du mariage, il peut très bien être intégré comme faisant partie d'une sexualité masculine acceptable et acceptée. Ce ne sont pas tant les pratiques effectives, insertives ou réceptives qui posent problème, mais plutôt l'affichage et la publicité dont ces pratiques font l'objet. Un individu que l'on définit comme zamel, parce qu'on le soupçonne de s'adonner à des pratiques sexuelles passives, et parce qu'il assume ou qu'on lui prête publiquement des attitudes et des comportements qui ne se conforment pas à la masculinité idéale, peut être réintégré en tant que «vrai homme» («rajul»). En revanche, pour un homme qu'on ne définit pas comme zamel, parce qu'il assume le rôle actif, et surtout parce qu'il s'affiche et il s'exprime, par ses attitudes corporelles, par son statut social et par sa présentation de soi dans l'espace public, en tant que « vrai homme », la question de la réintégration et de la stigmatisation ne se pose pas.

Tel a été le cas de Rachid. Il avait à l'époque 25 ans et était originaire du nord du Maroc. Il avait déménagé à Marrakech avec sa famille quelques années auparavant. Diplômé, ayant souvent voyagé en Europe, il travaillait depuis plusieurs années et appartenait à une famille de classe moyenne. Il n'était pas riche mais assez aisé pour pouvoir sortir en boîte de nuit. Il ne buvait pas, n'étant pourtant pas, religieusement, un pratiquant assidu. Il était respecté par ses amis en raison de sa discrétion et aussi parce qu'il approchait rarement des Européens. Par ailleurs, je ne l'ai jamais vu « draguer» 
quelqu'un dans un bar ou dans une boîte de nuit. En règle générale, il préférait faire ça sur l'Internet. Il avait eu plusieurs expériences de relations amoureuses et sexuelles avec d'autres garçons. Il venait de sortir d'une assez longue histoire d'amour avec un jeune garçon de sa ville natale. Ils avaient vécu leur relation d'amour sans entrave. Ils étaient, en fait, considérés par leurs familles respectives comme de bons amis. Ils s'étaient quittés pour des raisons d'incompatibilité de caractère et aussi parce que Rachid, ayant déménagé à Marrakech, ne supportait plus leur relation à distance. Jamais il ne m'a dit avoir subi une quelconque stigmatisation. Ayant des attitudes très masculines, cherchant à se définir à travers une grille taxinomique occidentale, il se disait ouvertement bisexuel. Je n'ai jamais su quelles étaient précisément ses préférences en termes de pratiques sexuelles, mais j'ai compris de ses discours qu'être actif ou passif ne lui posait pas un vrai problème.

Au moment où je l'ai connu à l'hiver 2005, il préparait à la fois son mariage et son départ pour les Pays-Bas. Rachid avait un très bon rapport avec sa famille, et il avait consenti à se marier avec une cousine éloignée vivant aux Pays-Bas, qui avait été choisie pour lui par ses parents. Le mariage était prévu pour la fin de l'été, au Maroc, et son départ pour l'Europe quelques temps après. Il connaissait à peine sa cousine, mais ne considérait pas son mariage comme une épreuve ou une violence. Il le voyait plutôt comme une opportunité d'émigration, et surtout de réalisation personnelle. Il voulait, en fait, construire une famille et avoir des enfants. Il pensait, en outre, que ses désirs pour les hommes pouvaient très bien se concilier avec une vie de famille. De plus, le fait d'émigrer aux Pays-Bas après le mariage lui laissait entrevoir une activité homoérotique encore plus épanouie et libre que celle qu'il avait eue jusqu'alors au Maroc.

La situation est, à l'inverse, très différente pour les garçons des classes supérieures ou moyennes. Pour eux, en général, le mariage demeure une espèce d'épée de Damoclès qu'ils redoutent et cherchent à repousser le plus longtemps possible. Ainsi, Redouan, âgé de 27 ans, issu d'un milieu riche de Casablanca et vivant à Paris depuis ses 18 ans pour ses études, que j'avais rencontré un soir à l'Étoile ${ }^{22}$, était très inquiet pour son avenir. Approchant la trentaine, il menait sa vie de gay parisien depuis plusieurs années, et n'avait jamais dit à ses parents qu'il était homosexuel, quoiqu'il pensait qu'ils s'en doutaient un peu. Il me raconta qu'il avait peur pour les années à venir parce que ses parents, mais surtout ses grands-parents, commençaient à faire pression sur lui pour qu'il se marie. Ce qui le sauvait pour le moment était le fait que son frère aîné de quelques années n’était pas encore marié, ce qui lui laissait encore quelque temps de tranquillité.

22. L'Étoile est un pseudonyme d'une boîte de nuit de Marrakech connue pour être un lieu de rencontre gay, ou gay-friendly. Elle faisait partie d'un complexe hôtelier plus ample destiné à accueillir un public européen. Pour une analyse plus complète des lieux de «drague » de l'homoérotisme et de l'homosexualité masculine, voir G. REBUCINI (2011). 
Le mariage, il n'en voulait pas. Il le considérait comme une trahison de ce qu'il était réellement, et il ne se voyait pas du tout faire ménage avec une femme. Il pensait que peut-être ses parents l'avaient compris mais que visà-vis du reste de sa famille, un jour ou l'autre, le problème de son mariage émergerait à la surface. Il espérait pouvoir continuer comme ça jusqu'à ce que « la mentalité change au Maroc ». Pour lui, le mariage ne représentait donc pas l'aboutissement de sa masculinité, mais plutôt une rupture vis-àvis de son identité homosexuelle. L'exclusivité homosexuelle de son identité n'aurait pas supporté l'imposition d'un mariage. D'un autre côté, il sentait que sa famille attendait de lui qu'il se marie un jour ou l'autre, et il ne voulait, mais surtout ne pouvait plus, rompre avec eux. Il se sentait bloqué, en situation de double contrainte: d'une part le respect de son identité sexuelle l'empêchait de satisfaire les volontés de ses parents et, d'autre part, il ne voulait pas les décevoir. Il avait ainsi décidé de laisser faire le temps et de ne pas s'inquiéter du futur pour le moment, jusqu'au jour où le problème serait posé comme inéluctable.

Si pour les jeunes des classes populaires, le mariage ne représente pas une rupture vis-à-vis de leur parcours et de leur identité, il n'en va pas de même pour les individus qui ont grandi dans un milieu occidentalisé. Dans les deux cas, l'identité masculine est mise en question de façon différente. Dans le premier cas, le mariage garantit, même si c'est d'une façon différée, la pleine acquisition des caractéristiques de la masculinité, mais dans l'autre, la masculinité est mise en danger par la même épistémologie occidentale que les jeunes appellent en secours, parce qu'elle inscrit l'acquisition d'une pleine masculinité dans la trajectoire d'une identité hétérosexuelle, à laquelle ils n'ont pas véritablement accès.

À ce point de notre analyse, il semble important de souligner que c'est l'agencement des diverses masculinités par rapport à la structure et à la signification de la famille qui devient l'élément discriminant dans les deux configurations sociales. Si, d'un côté, la famille demeure l'horizon constitutif de la construction de la personnalité et des possibilités et capacités d'agir de l'individu, de l'autre côté le modèle familial agit plutôt comme une entrave à l'épanouissement personnel et à l'agency des acteurs. Cela semble être en effet le résultat de la place différente que les liens familiaux entretiennent avec les deux épistémologies sexuelles que l'on a décrites.

Dans le modèle "genre/pratiques érotiques", dans le cadre duquel s'expriment les pratiques homoérotiques, parce que ces pratiques ne sont pas considérées comme une atteinte au bon déroulement de la téléologie du mariage, les hommes ont à leur disposition un plus grand éventail de choix possibles. Dans le rapport homoérotique, la possibilité d'accéder ou pas aux différentes pratiques, insertives ou réceptives, ne dépend pas de 
l'acceptation d'un modèle sexuel exclusif et identitaire qui départagerait les hommes entre homosexuels et hétérosexuels. Il semble plutôt que les hommes puissent gérer leurs pratiques érotiques en fonction de désirs qui ne dépendent pas forcément d'un agencement particulier de la personnalité « sexuellement » déterminée. Ces désirs seront davantage fonction d'autres aspects de la personne, comme une certaine relation à la construction de la masculinité hégémonique. Cette construction s'exprime dans un champ relationnel, comme dans le cas du rapport aux sentiments de dépendance et d'autonomie interpersonnelles, qui, comme on l'a vu, sont déterminants dans la généalogie et dans l'éducation à la masculinité. Ces sentiments ne sont pourtant pas relationnels dans le seul sens qu'ils sont en relation entre eux et se définissent mutuellement. Ils sont relationnels parce que pour s'actualiser et se concrétiser, ils nécessitent tout le système de relations sociales et un plus large réseau de connections, qui d'ailleurs se fondent sur la centralité institutionnelle et matérielle de la famille (qu'elle soit élargie ou nucléaire). En particulier, le sentiment d'autonomie, qui est celui qui exprime le plus l'aboutissement d'une masculinité mûre et hégémonique, ne doit pas être pensé dans les termes de l'individualisme libéral. Dans ce contexte socioculturel, l'autonomie personnelle est toujours entendue comme un concept relationnel. On est autonome, financièrement, intellectuellement, et moralement parce que l'on est capable de satisfaire les besoins et les attentes d'un réseau de relations, capacité qui seule peut confirmer cette position de privilège. Idéalement la construction de l'autonomie personnelle n'est pas tant l'aboutissement d'un individu que la mise en place de relations complexes dans un contexte social. L'autonomie n'a donc de sens que dans le cadre d'un tissage serré de liens interpersonnels que l'individu sera capable (ou qu'il aura l'occasion et la possibilité) de nouer avec les autres membres de sa communauté, à partir notamment de sa propre famille.

À l'inverse, la conception libérale de l'autonomie et de l'individu, qui est celle qui régit pour la plupart les expériences de vie et leurs interprétations dans le cas des identités homosexuelles et gay, est en opposition avec une structure de liens sociaux forts qui seraient déterminants dans la construction de la personne. La philosophie de l'individualisme, et donc, à partir de là, de la conception de l'autonomie de l'individu, fait une place importante à l'épanouissement de la personne notamment à travers un détissage des liens familiaux. La formation même de l'individu est conçue comme un long chemin vers l'autonomisation depuis sa propre famille et vers la construction de liens sociaux nouveaux qui dépendent toujours de l'intentionnalité de l'individu. L'autonomie individuelle dans ce contexte se veut donc fondamentalement en opposition avec sa famille d'origine. Ce n'est donc pas un hasard si, comme on l'a vu, les identités gay se fondent avant tout sur une anxiété vis-à-vis de la famille, et si le coming-out est en quelque sorte le moment et le signe par excellence de l'autonomisation de la personne homosexuelle ou gay. Le coming-out est, en effet, l'exemple le plus facilement identifiable du caractère fondé sur une épistémologie 
libérale des identités gay. Il est aussi le signe d'une certaine conception de l'autonomisation fondée sur une idée identitaire et individualiste de la personne.

Ce type de conception de la personne et de l'autonomie peut donc fonctionner dans un contexte où l'organisation générale de la société, et notamment de la famille et de la parenté, est fondée sur un modèle libéral. Dans une société dans laquelle, pour une bonne partie des individus, cette épistémologie libérale n'est pas l'horizon conceptuel et existentiel de référence (elle demeure néanmoins une des possibilités disponibles sinon envisageables), la famille reste le lieu et l'agencement institutionnel et affectif à partir duquel, et à l'intérieur duquel, les hommes trouvent les meilleures conditions et possibilités pour gérer, cultiver et même accrôtre leur propre capacité d'agir. Pour les hommes et les garçons qui se trouvent dans une telle situation existentielle, sortir de ce contexte social, couper les liens familiaux, s' «autonomiser» selon une acception libérale du terme, ce ne serait donc que perdre grandement en agency et en émancipation.

Dans les deux cas analysés plus haut, nous comprenons mieux maintenant que c'est le rapport avec la famille et le mariage (le mariage est l'institution reproduisant et maintenant les liens familiaux) qui est le point central de la différence entre les garçons étant dans un contexte d'épistémologie "genre/pratiques érotiques » et ceux s'étant formés à partir et dans une épistémologie identitaire homosexuelle. Dans le premier cas, les pratiques homoérotiques ne sont pas en opposition avec une bonne acquisition de la masculinité hégémonique, par le biais du mariage et du maintien des liens familiaux. Comme on l'a vu, ces pratiques peuvent aussi subsister après le mariage et elles ne sont pas des pratiques de «substitution».

Laboratoire d'anthropologie des institutions et des organisations sociales (LAIOS), EHESS-CNRS, Paris.

\section{BIBLIOGRAPHIE}

Abukhalil, A.

1993 «A Note on the Study of Homosexuality in the Arab/Islamic Civilization », Arab Studies Journal, I : 32-48.

ABu-Lughod, L.

1986 Veiled Sentiments : Honour and Poetry in a Bedouin Society, Berkeley, University of California Press.

Achehbar, S.

2007 «Événement. Choquant! La chasse à l’homo », TelQuel, 300, 1-7 décembre. 
ADAM, B.

1990 The Rise of a Gay and Lesbian Movement, Boston, Twayne Publications.

BERNICHI, L.

2007 «Ils ont bon dos les homos », Maroc Hebdo International, 769, 30 novembre6 décembre.

Boundiba, A.

1975 La sexualité en Islam, Paris, PUF.

Chauncey, G.

2003 [1994] Gay New York, 1890-1940, Paris, Fayard.

Chebel, M.

1988 L'esprit du sérail. Perversions et marginalités sexuelles au Maghreb, Paris, Lieu Commun.

ConNell, R. W.

1995 Masculinities, Cambridge (Mass.), Polity Press.

Connell, R. W. \& Messerschmidt, J. W.

2005 «Hegemonic Masculinity: Rethinking the Concept», Gender \& Society, 19 (829) : 829-859.

D'Emilio, J.

1993 « Capitalism and Gay Identity », in H. Abelowe, M. A. Barale \& D. HalPerin (eds.), The Lesbian and Gay Studies Reader, New York, Routledge : 467476.

DORLIN, E.

2006 La matrice de la race. Généalogie sexuelle et coloniale de la nation française, Paris, La Découverte.

DunNe, B.

1990 «Homosexuality in the Middle-East : an Agenda for Historical Research », Arab Studies Quaterly, 12 : 55-82.

DURAN, K.

1993 «Homosexuality and Islam », in A. SwidLer (ed.), Homosexuality and World Religions, Valley Forge, Trinity Press International.

EL-RouAYHEB, K.

2010 L'amour des garçons en pays arabo-islamique, Paris, EPEL [Édition originale, Before Homosexuality in the Arab-Islamic World, 1500-1800, Chicago, University of Chicago Press, 2005].

FEINBERG, L.

2006 «Islam Bashing Part of Racist War for Empire », Workers World, 8 février, $<$ http://www.workers.org/2006/world/islam-0216/>. 
Foucault, M.

1976 La volonté de savoir, Paris, Gallimard.

1984 «La généalogie de l'individu moderne. Questions et réponses », in H. DrEYFus \& P. RABINow (dir.), Michel Foucault. Un parcours philosophique, Paris, Gallimard : 242-263.

Gluckman, A. \& Reed, B.

1997 Homo Economics : Capitalism, Community, and Lesbian and Gay Life, New York, Routledge.

GREENBERG, S.

1988 The Construction of Homosexuality, Chicago, University of Chicago Press.

HALPERIN, D.

1990 One Hundred Years of Homosexuality and Other Essays on Greek Love, New York, Routledge [Édition française, Cent ans d'homosexualité et autres essais sur l'amour grec, Paris, EPEL, 2000].

HAMEL, C.

2002 «En milieu maghrébin, une question d'honneur», in R.-M. Lagrave, A. Gestin, É. LéPinard \& G. Pruvost (dir.), Dissemblances. Jeux et enjeux du genre, Paris, L'Harmattan : 37-50.

2003 « Maghreb », in L.-G. TIN (dir.), Dictionnaire de l'homophobie, Paris, PUF : 274-277.

Hammoudi, A.

2001 Maîtres et disciples. Genèse et fondements des pouvoirs autoritaires dans les sociétés arabes. Essai d'anthropologie politique, Paris, Casablanca, Maisonneuve \& Larose-Éd. Toubkal.

HAMrouch, M.

2007 «Affaire de Ksar El-Kébir : Prison ferme pour les accusés », Aujourd'hui le Maroc, 12 décembre.

HARAWAY, D. (DIR.)

2009 [1988] «Savoirs situés : la question de la science dans le féminisme et le privilège de la perspective partielle », in D. HARAWAY (dir.), Des singes, des cyborgs et des femmes. La réinvention de la nature, Paris, Éditions Jacqueline Chambon : 323-353 [Édition originale, «Situated Knowledge : The Science Question in Feminism and the Privilege of Partial Perspective », Feminist Studies, 14 (3) (1988: Fall) : 575-599].

HARITAWORN, J.

2008 «Loyal Repetitions of the Nation: Gay Assimilation and the "War on Terror" », DarkMatter, 3, numéro spécial « Postcolonial Sexuality », $<$ http://www.darkmatter101.org >.

Haritaworn, J., Tauqir, T. \& Erdem, E.

2008 «Gay Imperialism : Gender and Sexuality Discourse in the "War on Terror" ", in A. Kuntsman \& E. MiYAKe (eds.), Out of Place: Interrogating Silences in Queerness/Raciality, York, Raw Nerve Books : 71-96. 
HiLse Dwyer, D.

1978 Images and Self-Images, New York, Columbia University Press.

Howson, R.

2006 Challenging Hegemonic Masculinity, London-New York, Routledge.

KAPCHAN, D.

1996 Gender on the Market, Philadelphia, University of Pennsylvania Press.

KING, K.

2002 «"There Are No Lesbian Here": Lesbianism, Feminism, and Global Gay Formations », in A. Cruz-Malavé \& M. F. Manalansan IV (eds.), Queer Globalizations. Citizenship and the Afterlife of Colonialism, New YorkLondon, New York University Press : 33-45.

LACLAU, E.

2000 La guerre des identités : grammaire de l'émancipation, Paris, La Découverte.

LAGRANGe, F.

2008 Islam d'interdits, Islam de jouissance, Paris, Téraèdre.

DE LAURETIS, T.

2007 Théories queer et cultures populaires. De Foucault à Cronenberg, Paris, La Dispute («Le genre du monde»).

ManAlansan IV, M.

2003 Global Divas. Filippino Gay Man in the Diaspora, Durham-London, Duke University Press.

MASSAD, J.

2007 Desiring Arabs, Chicago, University of Chicago Press.

Mohanty, S. P.

2001 «Can Our Values Be Objective? On Ethics, Aesthetics, and Progressive Politics », New Literary History, 32 : 803-833.

Mosse, G. L.

1997 L'image de l'homme: l'invention de la virilité moderne, Paris, Éditions Abbeville.

Murray, S. O.

2000 Homosexualities, Chicago, University of Chicago Press.

Murray, S. O \& Roscoe, W.

1997 Islamic Homosexualities. Culture, History, and Literature, New York, New York University Press.

Pellat, C.

2002 «Liwât », in Encyclopedie de l'Islam, Leiden, Brill. 
Proth, B.

2002 Lieux de drague. Scènes et coulisses d'une sexualité masculine, Toulouse, Octarès.

PUAR, J. K.

2007 Terrorist Assemblages. Homonationalism in Queer Times, Durham-London, Duke University Press.

REBUCINI, G.

2009 Les masculinités au Maroc. Pour une anthropologie des genres et des sexualités dans la ville de Marrakech, Thèse de doctorat de troisième cycle, Paris, École des hautes études en sciences sociales.

2011 «Lieux de l'homoérotisme et de l'homosexualité masculine à Marrakech », L'Espace Politique, <http://espacepolitique.revues.org/index1830.html>.

SAÏD, E.

1978 Orientalism, London, Routledge \& Kegan Paul.

\section{Schmit, A.}

1992 « Different Approaches to Male-Male Sexuality/Eroticism from Morocco to Usbekistan », in A. Schmitt \& J. Sofer (eds.), Sexuality and Eroticism Among Males in Moslem Societies, New York, Haworth Press.

Wright, J. W. \& Rowson, E. K.

1997 Homoeroticism in Classical Arabic Litterature, New York, Columbia University Press.

RÉSUMÉ

Cet article présente une analyse des pratiques érotiques et des identités sexuelles entre hommes au Maroc et de leur relation aux masculinités. J'ai pu constater l'existence de deux épistémologies distinctes qui ordonnent les catégories définissant d'une part les pratiques érotiques - selon une généalogie locale - et, d'autre part, les identités sexuelles - dans un contexte de circulations transnationales. Ainsi, les pratiques érotiques entre hommes ne peuvent être décrites et comprises au prisme de la dichotomie homosexualité/hétérosexualité que pour une minorité de la population marocaine adoptant une épistémologie identitaire globalisée. À l'inverse de cette dernière, définie ici comme "système de genre/identités sexuelles", l'épistémologie locale " système de genre/pratiques érotiques » ne rentre pas en opposition avec une certaine construction de la masculinité hégémonique fondée sur le mariage et la procréation. 


\section{ABSTRACT}

Hegemonic Masculinities and "Sexualities" Among Men in Morocco. Between Local Configurations and Globalization of Gender and Sexual Categories. - This article presents an analysis of erotic practices and sexual identities between men in Morocco in relation to masculinities. I have found two distinct epistemologies that order the categories defining on one hand the erotic practices, with a local genealogy, and on the other hand the sexual identities inscribed in a context of transnational circulations. Thus, the erotic practices between men can be described and understood through the prism of the dichotomy between homosexuality/heterosexuality just for a minority of the Moroccan population. Moreover, contrary to the globalized epistemology of identities, defined here "gender/sexuality system", the local epistemology, called here "gender/erotic practices system", does not fit as opposed to a certain Moroccan construction of hegemonic masculinity, based on marriage and procreation.

Mots-clés/Keywords: Maroc, globalisation, homosexualité masculine, masculinités, pratiques érotiques/Morocco, globalization, male homosexuality, masculinities, erotic practices. 